\title{
Turbulence, waves, and jets in a differentially heated rotating annulus experiment
}

\section{Citation}

Wordsworth, R. D., P. L. Read, and Y. H. Yamazaki. 2008. "Turbulence, Waves, and Jets in a Differentially Heated Rotating Annulus Experiment." Physics of Fluids 20 (12): 126602. doi:10.1063/1.2990042. http://dx.doi.org/10.1063/1.2990042.

\section{Published Version}

doi:10.1063/1.2990042

\section{Permanent link}

http://nrs.harvard.edu/urn-3:HUL.InstRepos:27846858

\section{Terms of Use}

This article was downloaded from Harvard University's DASH repository, and is made available under the terms and conditions applicable to Open Access Policy Articles, as set forth at http:// nrs.harvard.edu/urn-3:HUL.InstRepos:dash.current.terms-of-use\#OAP

\section{Share Your Story}

The Harvard community has made this article openly available.

Please share how this access benefits you. Submit a story.

\section{Accessibility}




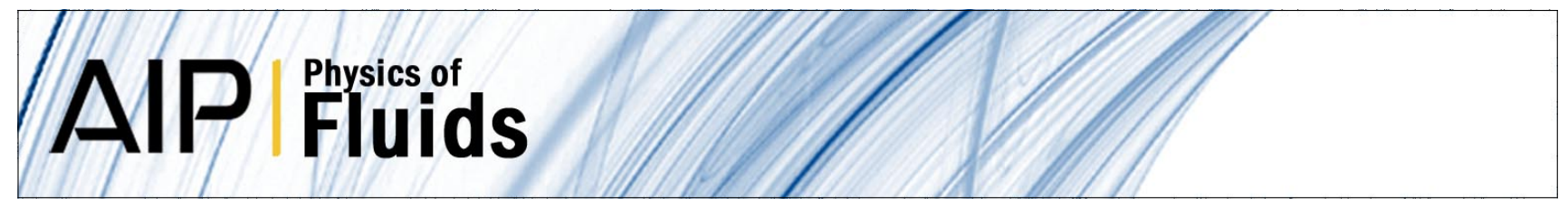

\section{Turbulence, waves, and jets in a differentially heated rotating annulus experiment}

R. D. Wordsworth, P. L. Read, and Y. H. Yamazaki

Citation: Phys. Fluids 20, 126602 (2008); doi: 10.1063/1.2990042

View online: http://dx.doi.org/10.1063/1.2990042

View Table of Contents: http://pof.aip.org/resource/1/PHFLE6/v20/i12

Published by the American Institute of Physics.

\section{Related Articles}

A characteristic function to estimate the longitudinal dispersion coefficient in surface water flows over porous media

Phys. Fluids 24, 046602 (2012)

Frequency and damping of non-axisymmetric surface oscillations of a viscous axisymmetric liquid bridge Phys. Fluids 24, 042103 (2012)

Nonlinear resonance in barotropic-baroclinic transfer generated by bottom sills

Phys. Fluids 24, 046601 (2012)

An eddy viscosity model for two-dimensional breaking waves and its validation with laboratory experiments

Phys. Fluids 24, 036601 (2012)

Microdroplet oscillations during optical pulling

Phys. Fluids 24, 022002 (2012)

\section{Additional information on Phys. Fluids}

Journal Homepage: http://pof.aip.org/

Journal Information: http://pof.aip.org/about/about_the_journal

Top downloads: http://pof.aip.org/features/most_downloaded

Information for Authors: http://pof.aip.org/authors

\section{ADVERTISEMENT}

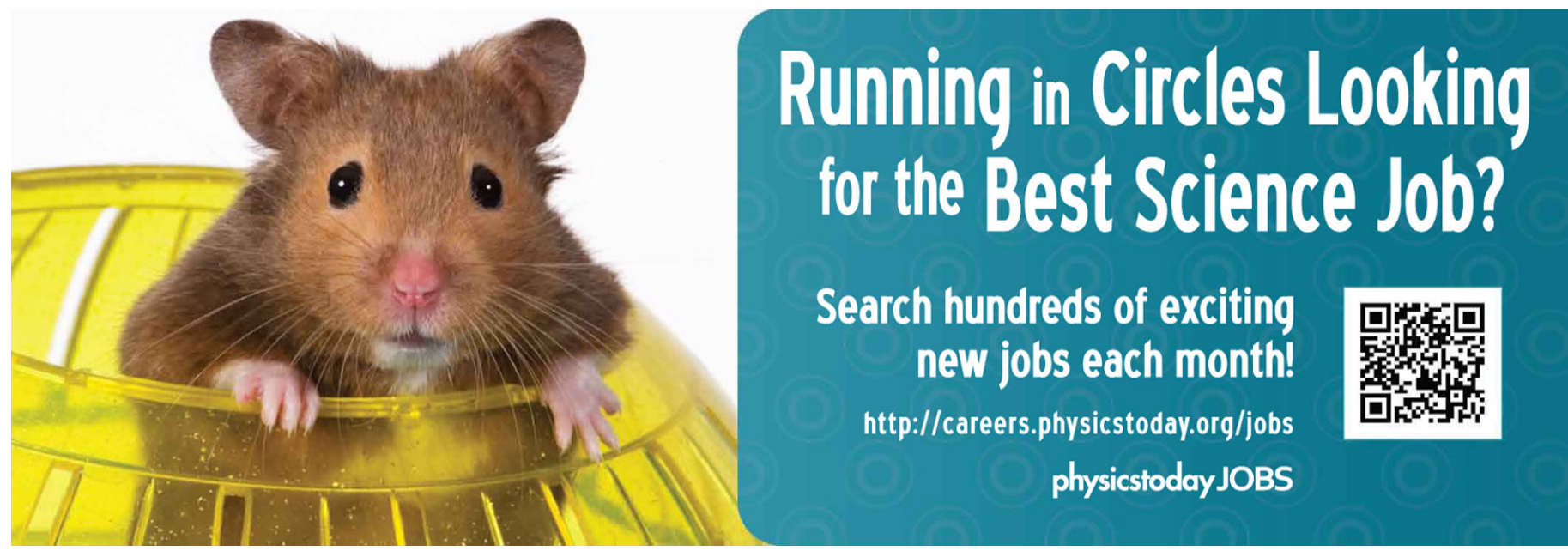




\title{
Turbulence, waves, and jets in a differentially heated rotating annulus experiment
}

\author{
R. D. Wordsworth, ${ }^{\text {a) }}$ P. L. Read, and Y. H. Yamazaki \\ Atmospheric, Oceanic and Planetary Physics, Clarendon Laboratory, Department of Physics, \\ University of Oxford, Parks Road, Oxford OX1 3PU, United Kingdom
}

(Received 16 March 2008; accepted 15 August 2008; published online 8 December 2008)

\begin{abstract}
We report an analog laboratory study of planetary-scale turbulence and jet formation. A rotating annulus was cooled and heated at its inner and outer walls, respectively, causing baroclinic instability to develop in the fluid inside. At high rotation rates and low temperature differences, the flow became chaotic and ultimately fully turbulent. The inclusion of sloping top and bottom boundaries caused turbulent eddies to behave like planetary waves at large scales, and eddy interaction with the zonal flow then led to the formation of several alternating jets at mid-depth. The jets did not scale with the Rhines length, and spectral analysis of the flow indicated a distinct separation between jets and eddies in wavenumber space, with direct energy transfer occurring nonlocally between them. Our results suggest that the traditional "turbulent cascade" picture of zonal jet formation may be an inappropriate one in the geophysically important case of large-scale flows forced by differential solar heating. (c) 2008 American Institute of Physics.
\end{abstract}

[DOI: $10.1063 / 1.2990042$ ]

\section{INTRODUCTION}

Turbulent motion is ubiquitous in the atmospheres of all gas giant planets and most terrestrial planets in our Solar System. The primary energy source for the turbulence is usually either solar or internal heating; in general, planetary atmospheres are almost always far from thermal equilibrium with their surroundings.

On very large scales, atmospheric motion is usually strongly constrained by vertical stratification and the Coriolis effect, and hence is nearly geostrophic away from equatorial regions of the planet. Geostrophically turbulent flow behaves dramatically differently from the "classical" threedimensional isotropic case, ${ }^{1}$ and its importance is such that it is intensively studied in the fluid-dynamical literature.

One of the most insightful early results on the subject was due to Fjortoft, ${ }^{2}$ who demonstrated that for any nonlinear triad interaction in two-dimensional (2D) flows, energy is transferred preferentially to smaller wavenumbers. The fundamental basis for 2D and geostrophic "inverse energy cascade" theories of turbulence, this result broadly explains the tendency for atmospheric motion to be dominated by largescale coherent structures such as the jets and vortices of the gas giants, despite the fact that at least some of the forcing is likely to be occurring on far smaller scales. ${ }^{3}$

Another constraint on planetary circulation that strongly affects the nature of large-scale flows is the variation in Coriolis effect with latitude or planetary $\beta$-effect. The development of geostrophic turbulence on an idealized $\beta$-plane was studied by Rhines, who predicted in a seminal paper ${ }^{4}$ that planetary wave motion arising from the $\beta$-effect would alter the inverse cascade of energy at low wavenumbers, causing the transfer of energy into the zonal modes. This

$\overline{\left.{ }^{a}\right) \text { Electronic mail: robin@atm.ox.ac.uk. }}$
"Rhines effect" is frequently cited as an explanation for the zonal jets observed in the atmospheres of Jupiter and Saturn.

While insightful, Rhines' work was essentially heuristic and could not provide a detailed dynamical explanation of the process. As a result, barotropic $\beta$-plane jet formation has been the subject of intensive numerical study by many researchers (e.g., Refs. 5-7). Notably, Vallis and Maltrud ${ }^{8}$ studied decaying unforced turbulence and suggested that planetary wave motion should dominate inside a dumbbellshaped region aligned along the axis of the east-west wavenumber $k_{x}$ in spectral space. From their simulations, they found that energy transfer slowed dramatically in this region, and instead proceeded mainly toward the zonal modes. However, they also noted that there was no a priori justification for zonal energy to peak at the Rhines scale.

More recently, Sukoriansky et al. ${ }^{9}$ produced an empirical theory for the spectral scaling observed in 2D simulations of turbulent flows on the surface of a rotating sphere. Galperin et $a l .{ }^{10}$ noted further properties of these idealized systems, including the importance of the large-scale damping mechanism in determining the steady-state jet structure. Finally, a recent experiment at the large-scale Coriolis facility ${ }^{11}$ also reproduced $\beta$-plane multiple jet formation in a convectively driven laboratory flow.

In real atmospheres, the role of vertical structure on the dynamics is generally non-negligible. In particular, direct energy input to the system can occur through baroclinic instability (ultimately due to differential solar heating with latitude). Salmon ${ }^{12}$ argued that for baroclinically forced turbulence, the internal deformation radius can be treated as an "energy input scale" to the quasi-2D barotropic flow, which otherwise behaves more or less as it would in the generic small-scale forcing case. In particular, a barotropic inverse cascade is generally still expected to transfer kinetic energy from small to large scales. 
As Salmon's argument is based only on conservation laws and an irreversibility principle, it makes quite unambiguous predictions, but only about the general direction of energy transfer. Further theoretical progress has proved difficult, not least because for many real flows the deformation radius is not much smaller than the observed jet width. In this situation standard cascade-type arguments, which are dependent on the existence of well-defined inertial ranges in spectral space, are of dubious validity.

Some numerical simulations of baroclinically forced turbulence have been carried out. Panetta ${ }^{13}$ simulated two-layer $\beta$-plane turbulence in a doubly periodic domain and found that equivalent barotropic jets with width proportional to the Rhines scale formed, with the system reaching a steady state over a time-scale longer than that predicted by any simple combination of model parameters. However, the computational power needed to simulate fully three-dimensional turbulent flows for long time periods means that this problem has not been well studied, in general. In particular, large uncertainties remain concerning the exact nature of energy transfer from baroclinic to barotropic modes, as well as that between eddies and the mean zonal flow.

We used a differentially heated rotating annulus to generate baroclinically forced turbulence in the laboratory. First developed by Hide,${ }^{14}$ the "baroclinic annulus" is intended as an extremely simple prototype of the motion of real planetary atmospheres. In essence, the idea behind the experiment is to mimic the effects of tropical heating and polar cooling on a midlatitude air mass by heating and cooling, respectively, the outer and inner walls of an annulus filled with some working fluid. It has now been extensively studied in a wide range of configurations-for a comprehensive review of important previous results, see, e.g., Ref. 15. However, due to the difficulty involved in (a) pushing the system into a geostrophically turbulent state and (b) acquiring detailed data on the flow when such a state is reached, previous research has tended to focus on the weakly nonlinear behavior of the system.

Here, we use new data acquisition techniques and a large annulus setup that allows us to investigate more strongly turbulent flows in more detail than has previously been possible in the laboratory or via numerical simulation. We focus on the steady-state properties of the system in the turbulent regime and examine both flat and sloping boundary cases. The latter case has previously been studied in detail only in the weakly nonlinear and chaotic regimes. ${ }^{16}$ One past investigation involving internally heated fluids in a slightly different setup began to show evidence of zonal jet formation at high rotation rates. ${ }^{17}$ However, it was not possible in that study to investigate the behavior of the jets in detail.

The aim of this paper, therefore, is to elucidate the dynamics of the turbulent jet formation regime. In Sec. II we describe the experimental apparatus. In Sec. III an overview of the results for both flat and sloping bottom boundary experiments is given. General features of the flow are discussed first, followed by progressively more detailed analyses of jet and eddy dynamics. Finally, in Sec. IV we discuss some conclusions from our results, including implications for turbulent flows observed in real planetary atmospheres.

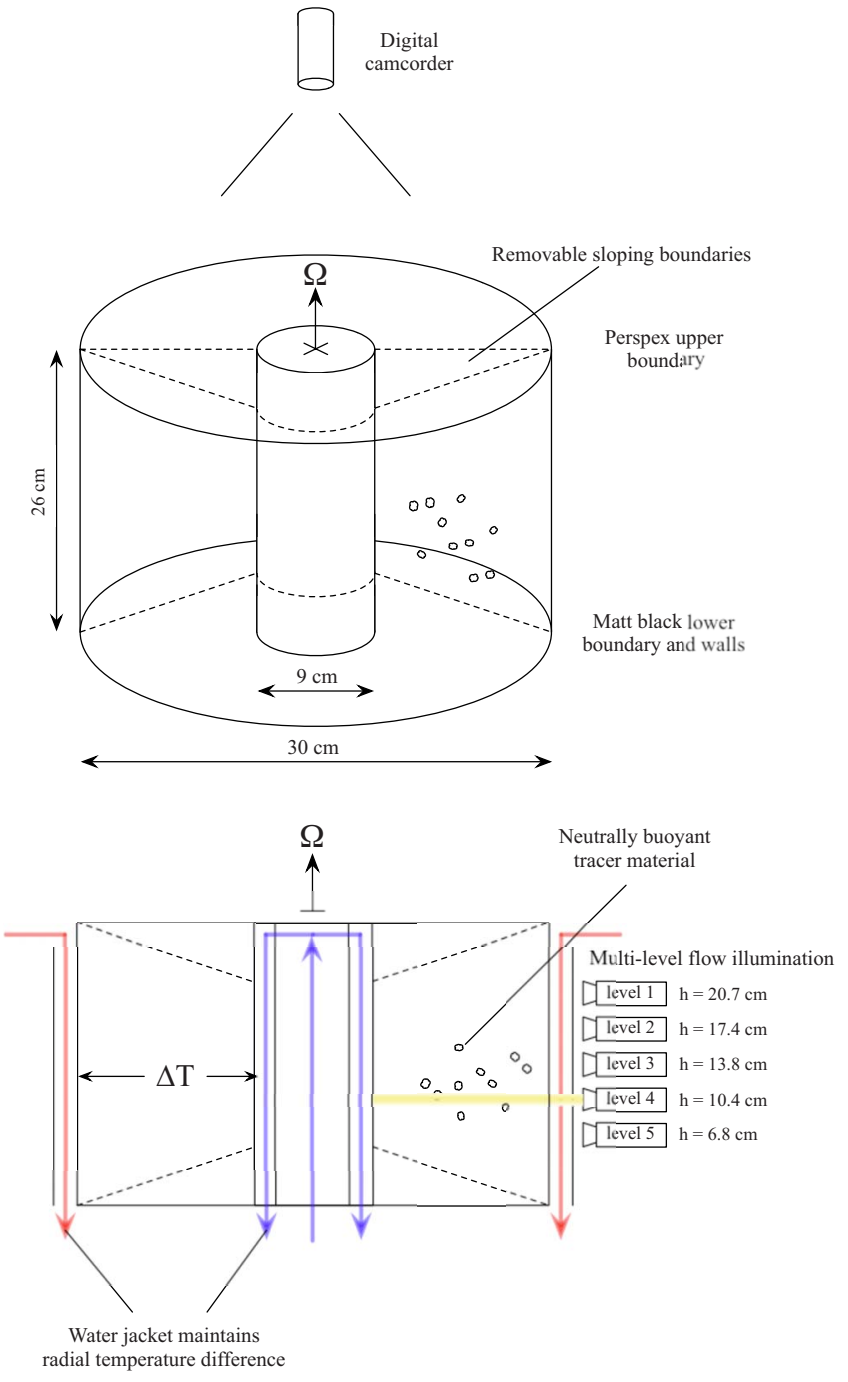

FIG. 1. (Color online) Schematic of the apparatus used for all experiments. A temperature controlled water jacket was used to maintain the difference $\Delta T$ between inner and outer walls.

\section{APPARATUS}

Our experimental setup is as follows. An annulus filled with a working fluid is set on the center of a rotating turntable, and its inner and outer walls are cooled and heated, respectively, in order to maintain a constant temperature difference between the two (see Fig. 1). By the thermal wind equations (see, e.g., Ref. 1), in a rotating frame this temperature difference causes a vertically sheared zonal velocity profile. When the rotation rate of the turntable is increased above a certain critical value, this axisymmetric flow then becomes hydrodynamically unstable and can evolve into a wide variety of steady or chaotic flow states. The state of the system is principally determined by two dimensionless numbers: the Taylor number

$$
T a \equiv \frac{4 \Omega^{2}(b-a)^{5}}{\nu^{2} d} \approx \frac{[2 \Omega \times \mathbf{u}]^{2}}{\left[\nu \nabla^{2} \mathbf{u}\right]^{2}},
$$

which is a ratio between Coriolis and viscous effects, and the Hide number 
TABLE I. General experimental parameters.

\begin{tabular}{llc}
\hline \hline Radius of inner cylinder & $a$ & $4.5 \mathrm{~cm}$ \\
Radius of outer cylinder & $b$ & $14.3 \mathrm{~cm}$ \\
Annulus depth (flat) & $d$ & $26 \mathrm{~cm}$ \\
Annulus mean depth (sloping) & $\bar{d}$ & $21.5 \mathrm{~cm}$ \\
Gravitational acceleration & $g$ & $9.81 \mathrm{~m} \mathrm{~s}^{-2}$ \\
Sloping boundary angle & $\delta$ & $22^{\circ}$ \\
Kinematic viscosity of fluid A & $\nu$ & $2.04 \times 10^{-6} \mathrm{~m}^{2} \mathrm{~s}^{-1}$ \\
Kinematic viscosity of fluid B & $\nu$ & $1.11 \times 10^{-6} \mathrm{~m}^{2} \mathrm{~s}^{-1}$ \\
Volumetric expansion coefficient & $\alpha$ & $3.16 \times 10^{-4} \mathrm{~K}^{-1}$ \\
\hline \hline
\end{tabular}

$$
\Theta \equiv \frac{\alpha g d \Delta T}{\Omega^{2}(b-a)^{2}},
$$

which is essentially a ratio between buoyancy and Coriolis effects or "thermal Rossby number." 14 Definitions and values of all general experimental parameters are given in Table I.

Previous studies have shown that the transition to turbulent flow occurs at large Taylor and small Hide numbers. We used an apparatus with a relatively large gap width $(b-a)$, which at high rotation rates allowed us to reach Taylor numbers an order of magnitude greater than had previously been used in experimental studies with sloping boundaries. ${ }^{17}$

As mentioned in Sec. I, the top and bottom boundaries of the annulus can be either flat or sloping. In the sloping case, motion of fluid columns in the radial direction is constrained due to conservation of local angular momentum. For a barotropic fluid (no variation in velocity with depth), the effects of sloping topography are the same as those of the planetary $\beta$-effect. A "topographic beta parameter"17 can be defined

$$
\beta=\frac{4 \Omega \tan \delta}{\bar{d}},
$$

where $\bar{d}$ is the mean depth of the fluid with boundaries included and $\delta$ is the angle of the slope. Note that for simplicity, we use the maximum depth $d$ in all other dimensionless numbers, in order to make direct comparison between flat and sloping experiments easier.

For a baroclinic (vertically sheared) flow, the analogy is no longer exact, as sloping boundaries also modify the zonally symmetric temperature field and hence the growth rate of instabilities. However, previous studies ${ }^{16}$ have shown that the qualitative aspects of flow behavior are not strongly affected by this change.

The internal deformation radius is another important quantity in baroclinically forced turbulence, as it is the scale at which energy is optimally transferred from baroclinic to barotropic modes. Here it is defined as

$$
L_{d}=\frac{\sqrt{\alpha g d \Delta T_{z}}}{\Omega},
$$

where $\Delta T_{z}$ is the vertical temperature difference. Although we do not have temperature information for these experiments, an upper bound on the deformation radius $L_{\max }>L_{d}$ can be derived by replacing $\Delta T_{z}$ in Eq. (4) with the imposed horizontal temperature gradient $\Delta T$. As will be seen, this upper limit is extremely useful for analyzing the properties of the turbulent flow regimes.

We used flow visualization to acquire information on the interior dynamics of the fluid. Neutrally buoyant tracer particles of radius of 350-500 $\mu \mathrm{m}$ were suspended in the fluid and alternately illuminated by thin $(\approx 0.5 \mathrm{~cm})$ light sheets at five different depths. For most experiments we chose a $82.5 \%-17.5 \%$ water/glycerol mix (fluid A) as the working fluid, in order to match the density of the tracer particles, $\rho=1.043 \mathrm{~g} \mathrm{~cm}^{-3}$. Some experiments were also performed with a $\mathrm{NaCl}$ salt solution (fluid $\mathrm{B}$ ): in this case the density was the same, but the fluid viscosity was only $\nu=1.11$ $\times 10^{-6} \mathrm{~m}^{2} \mathrm{~s}^{-1}$, allowing a greater Taylor number and hence a potentially more turbulent flow at the same rotation speed as in the previous case.

The flow was imaged from above by a digital camcorder (720 $\times 576$ resolution), which was connected to a small computer placed on the turntable during experiments. The turntable computer was then controlled remotely via a wireless ethernet connection with a second computer in the laboratory frame, allowing data acquisition and control without the need for slip rings or similar mechanical methods.

Raw images of the flow were converted to velocity fields by use of correlation imaging velocimetry software (CIV). ${ }^{18}$ In brief, CIV works by comparing images of the flow with a given time separation, tracking the displacement, rotation, and shear of image texture within small (typically $10 \times 10$ pixel) boxes. Unlike more traditional particle image velocimetry approaches, it does not need to resolve the motion of individual tracer particles, and hence is well suited to the analysis of flows with a large degree of spatial scale variation. For all data sets reported here, the Cartesian grids produced by CIV were interpolated onto $24 \times 144(r, \theta)$ polar grids before analysis.

The digital camcorder used a MINIDV format, which compresses images before storing them. We tested the error introduced by this by taking raw demonstration flow images from the CIV website, http://www.civproject.org, and artificially compressing them using the same algorithm as is used for the MINIDV video format. Both original and compressed images were then processed in CIV, and the resulting velocity fields compared. The difference in total kinetic energy between the two was less than $1 \%$. However, fluctuations due to image compression error tended to increase with wavenumber, reaching $10 \%$ at $k_{e}=0.1 \mathrm{rad} \mathrm{pixel}^{-1}$. In the spectral analysis of Sec. III B, therefore, we limit our diagnostics to modes with wavenumber smaller than this value.

In the case of sloping top and bottom boundaries, the slope on the top perspex boundary causes a slight deviation of light rays traveling from the working fluid to the camcorder because of the differing refractive indices of air, perspex, and the working fluid. To counteract this effect, the perspex boundary also had a slight slope $\left(\delta_{\text {top }}=3.5^{\circ}\right)$ on its upper surface. We also checked view distortion by photographing concentric black and white rings in the tank at different depths and found that the maximum error at the inner and outer walls was of order a few pixels only. This was judged sufficiently small to be neglected in the analysis.

We alternated the illumination of the five depth levels 


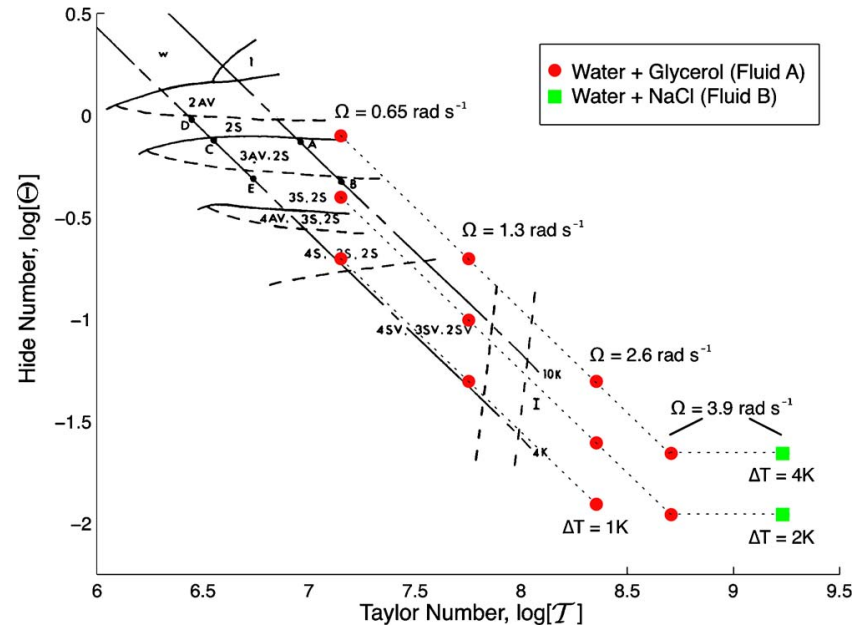

FIG. 2. (Color online) Logarithmic parameter space diagram showing the positions of all experiments performed as a function of Taylor and Hide number $(\mathcal{T}$ vs $\Theta)$. Also shown in black is a regime diagram derived from a previous experimental study (see Ref. 19, for details).

using a simple oscillation circuit with a manually adjustable time delay. This allowed us to derive quasisimultaneous horizontal velocity fields for the fluid at different depths for a wide range of flow regimes. The time delay between levels in the experiments was between 3 and $6.5 \mathrm{~s}$, depending on the observed flow velocity. The delay was chosen to be long enough to allow high quality CIV analysis, but short enough to allow quasisimultaneous visualization of the flow field. In all cases, it was smaller than (a) the minimum eddy turnover time and (b) the period of the fastest observed planetary waves (see Sec. III B).

To calculate barotropic modes for the spectral calculations, we interpolated multilevel data linearly in time between frames before vertically averaging. For a fully rigorous modal decomposition, weighting by level is required due to the variation in the interior temperature gradient $T^{\prime}(z)$ with depth. However, even for unrealistically high vertical temperature changes, this weighting deviates only by an extremely small amount from unity, and the error due to this approximation was neglected.

Spectra were also produced from mid-depth data and compared with the vertically averaged velocity fields. The difference between the two was small, suggesting that middepth fields were a good proxy for the interpolated barotropic data. We use the vertically averaged fields in Sec. III B. Finally, the scientific software MATLAB was used for all further data analysis and diagnostics. Where necessary, the details of more complicated analyses performed are discussed in the next section.

\section{RESULTS}

As mentioned in Sec. II, the experiments presented here investigate a parameter space region of high Taylor and low Hide number. The locations in parameter space of all experiments performed are shown in Fig. 2. Also shown, for comparison, is a regime diagram from a previous investigation in a smaller apparatus with similar aspect and radius ratios. ${ }^{19} \mathrm{In}$

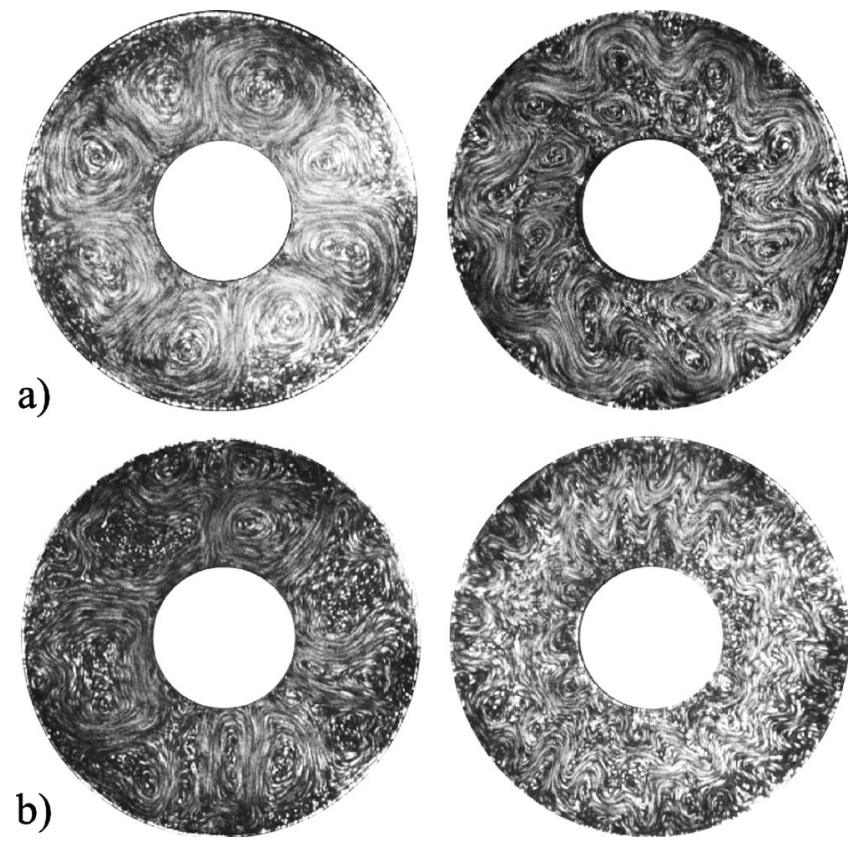

FIG. 3. Streak image comparison of the mid-depth flow in flat (left) and sloping (right) boundary cases for (a) low rotation rate $\Omega=1.3 \mathrm{rad} \mathrm{s}^{-1}$ and (b) high rotation rate $\Omega=3.9 \mathrm{rad} \mathrm{s}^{-1}$ with $\Delta T=2 \mathrm{~K}$.

this investigation, we varied rotation rate and temperature difference from $\Omega=0.65$ to $3.9 \mathrm{rad} \mathrm{s}^{-1}$ and $\Delta T=1$ to $4 \mathrm{~K}$.

As the principal interest of this investigation was the (statistically) steady-state properties of the flow, each experiment was run for $2 \mathrm{~h}$, with data collection occurring in the second hour only. The Ekman spinup time, defined as $\tau_{\mathrm{Ek}}=d / \sqrt{2 \nu \Omega}$, varies between 160 and $65 \mathrm{~s}$ for the experiments presented here: hence between 22 and 55 spinup times passed before data collection. Furthermore, after CIV analysis we plotted total energy and enstrophy of the flow as a function of time for each data set, and found that in all cases, these quantities did not exhibit any significant monotonic trend over the observed period.

Figure 3 shows streak images of the flow for flat and sloping boundary experiments at (a) low and (b) high rotation rates. Each image was taken after approximately $30 \mathrm{~min}$ of evolution time, with an averaging time of $20 \mathrm{~s}$.

The streak images in Fig. 3(a) are from experiments where the rotation rate was low enough for the flow to be chaotic rather than fully turbulent, at least in the flat boundary case. There, the flow appears to have been in a structurally vacillating "wavenumber 4 " state $(4 \mathrm{SV})$, which is expected, given the parameter space diagram recorded in Ref. 19 (Fig. 2). In the sloping case, a wavy jet close to the outer boundary is apparent, with the inner half of the channel dominated by a chain of moving vortices.

The streak images in Fig. 3(b) show more complicated behavior. The flows in both experiments were fluctuating fairly rapidly: the flat boundary case, in particular, exhibits varying radial and azimuthal eddy motion, with no single wavenumber dominating. In the sloping case, the large-scale domain-spanning eddies were replaced by apparent wavelike motion and weak zonal jets. The bottom-right image in Fig. 3 is somewhat reminiscent of the streak images reported pre- 


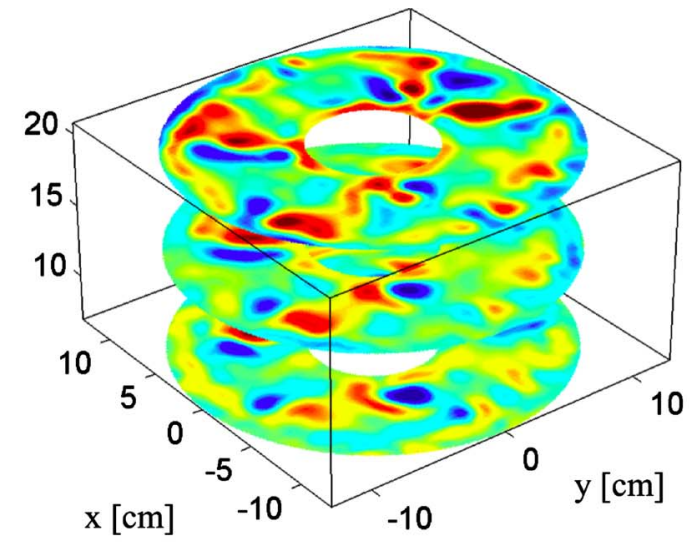

a)

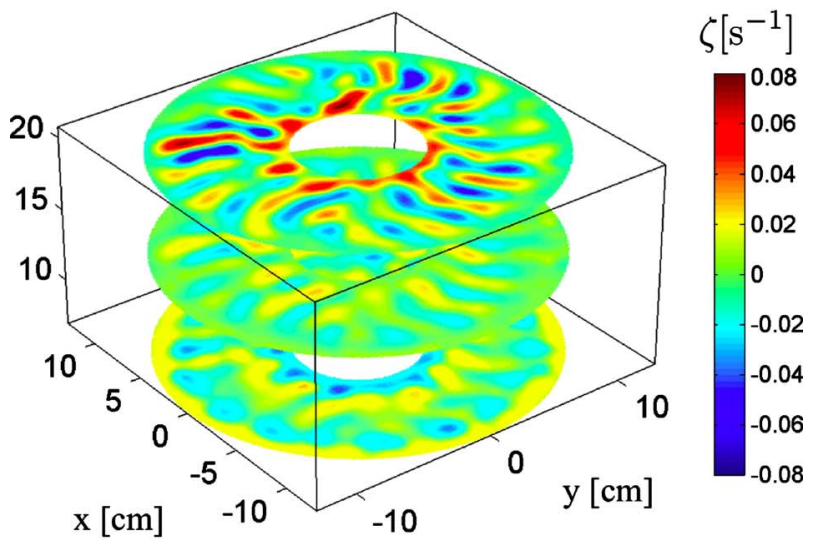

b)

FIG. 4. (Color online) Multilevel snapshot of vertical vorticity component at levels 1,3 , and 5 , time $t=3600 \mathrm{~s}$ for (a) flat boundary and (b) sloping boundary experiments with $\Delta T=2 \mathrm{~K}$ and $\Omega=3.9 \mathrm{rad} \mathrm{s}^{-1}$.

viously in a quite different setup $;^{20}$ there, a rotating parabolic dish was heated from below and allowed to cool convectively at its upper surface. However, little quantitative velocity field information was derived for that experiment, making further comparison difficult.

Figure 4 shows CIV-derived contour plots of nearinstantaneous vertical vorticity component $\zeta=r^{-1}\left(\partial_{r}\left(r u_{\theta}\right)\right.$ $\left.-\partial_{\theta} u_{r}\right)$ at three different depths in the fluid for the same experiments as displayed in Fig. 3(b). Three-dimensional visualization of this type helps to highlight the dramatic difference between the flow in the flat and sloping boundary cases-note, in particular, the pronounced wavelike appearance of Fig. 4(b). Animations of the vorticity field showed that the wave crests were traveling westward, as expected for disturbances that are qualitatively similar to atmospheric planetary waves. In Sec. III B, we discuss a temporal spectral analysis of the velocity field data that shows that much of this flow was indeed dominated by wavelike motion.

A selection of relevant length scales as a function of Taylor number for all the sloping boundary experiments is plotted in Fig. 5. The Rhines scale, defined as $L_{\mathrm{Rh}}=\sqrt{U / \beta}$, was calculated using a time and volume averaged value for the root mean square flow speed $U$. The jet scale was estimated by Fourier transforming the zonal, temporal, and vertical means of the azimuthal velocity $\left\langle\overline{u_{\theta}} \theta_{z}\right\rangle$ in radius. The
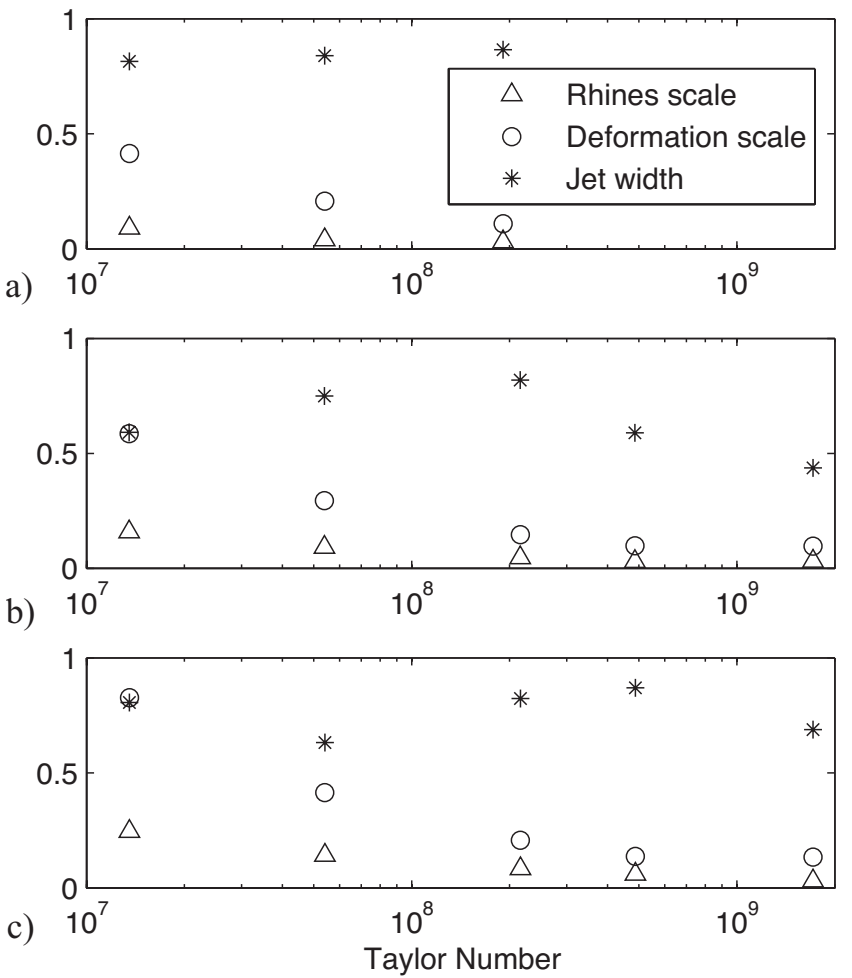

FIG. 5. Relevant length scales in units of channel width $(b-a)$ as a function of Taylor number for (a) $\Delta T=1 \mathrm{~K}$, (b) $\Delta T=2 \mathrm{~K}$, and (c) $\Delta T=4 \mathrm{~K}$ sloping boundary runs.

location of the peak of the resulting power spectrum was then taken to be the characteristic jet wavenumber, with jet scale the inverse $L_{\mathrm{jet}}=\pi / k_{\mathrm{jet}}$. This analysis was performed on both midlevel and vertically averaged data, but the difference between the two was found to be extremely small.

The horizontal buoyancy scale (upper limit on deformation radius) decreased with Taylor number, becoming less than one-tenth of the channel width for the highest rotation rate experiments. The Rhines scale for the sloping boundary runs also decreased with Taylor number, primarily because $\beta$ increased with $\Omega$. Neither length scales were good quantitative predictors for the observed jet width, although we note that for Taylor numbers greater than $\mathcal{T} \approx 2 \times 10^{8}$ for the $\Delta T=2 \mathrm{~K}$ runs, all three scales were at least decreasing in the same direction.

In the $\Delta T=1 \mathrm{~K}$ experiments, the observed flow was generally too weak to allow accurate velocity field measurement. In the $\Delta T=4 \mathrm{~K}$ experiments, we observed single eastward jets only, quite possibly because the deformation radius was too large to allow multiple jet formation. From here, therefore, we focus our analysis on the dynamically most interesting $\Delta T=2 \mathrm{~K}$ cases.

In Fig. 6, the instantaneous mid-depth vorticity (left) and zonal velocity $u_{\theta}=r \dot{\theta}$ (right) after $1.5 \mathrm{~h}$ are plotted for a range of such experiments. In the high rotation flat boundary experiment [Fig. 6(a)] both fields appear unstructured, with no evidence of coherent zonal structure formation. In the sloping boundary cases, jet formation at high rotation rates is clearly visible in the zonal velocity plots; the "fluid B" experiment, in particular, has a clear 2-3 jet structure 

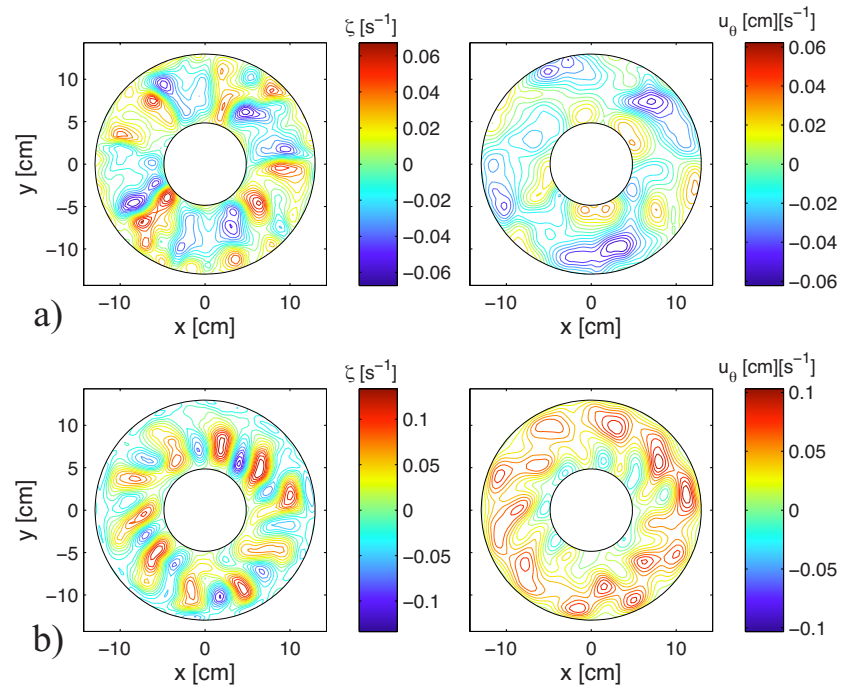

$\mathrm{u}_{\theta}[\mathrm{cm}]\left[\mathrm{s}^{-1}\right]$
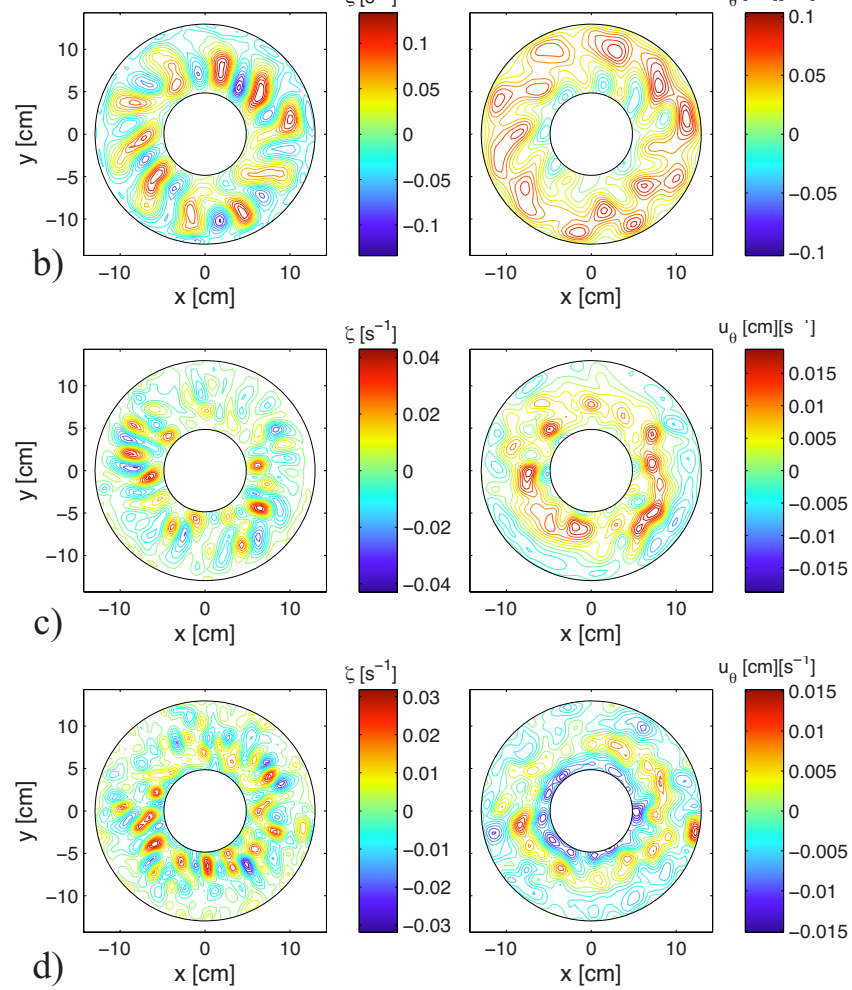

FIG. 6. (Color online) Snapshots of mid-depth vorticity and zonal velocity for experiments with constant temperature difference $\Delta T=2 \mathrm{~K}$. The cases are (a) flat boundary $\Omega=3.9 \mathrm{rad} \mathrm{s}^{-1},[(\mathrm{~b})$ and (c)] sloping boundary $\Omega=1.3,3.9 \mathrm{rad} \mathrm{s}^{-1}$, and (d) same rotation rate as (c) but with low viscosity working fluid $\mathrm{B}$.

[Fig. 6(d)]. It is most interesting that regions where the jets are strong tend to correspond to those where wave activity, as visible in the instantaneous vorticity fields, is highest. Also clearly apparent is the increase in wavenumber (both azimuthal and radial) of the vorticity fields with rotation. We examine these issues further in the next two sections.

\section{A. Eddy-mean flow interaction}

The effect of eddies, be they wavelike or turbulent, on zonal mean quantities, can be written in terms of the averaged correlation of the various eddy fields. In this section we examine the eddy (angular) momentum and heat fluxes, $\left\langle\overline{u_{r}^{\prime} u_{\theta}^{\prime}}\right\rangle$ and $\left\langle\overline{u_{r}^{\prime} T^{\prime}}\right\rangle$, and compare them to the observed timeaveraged zonal flow profiles.

Temperature information was derived in an indirect way via the quasigeostrophic approximation. First, streamfunction $\psi^{\prime}$ was derived numerically from the eddy vorticity
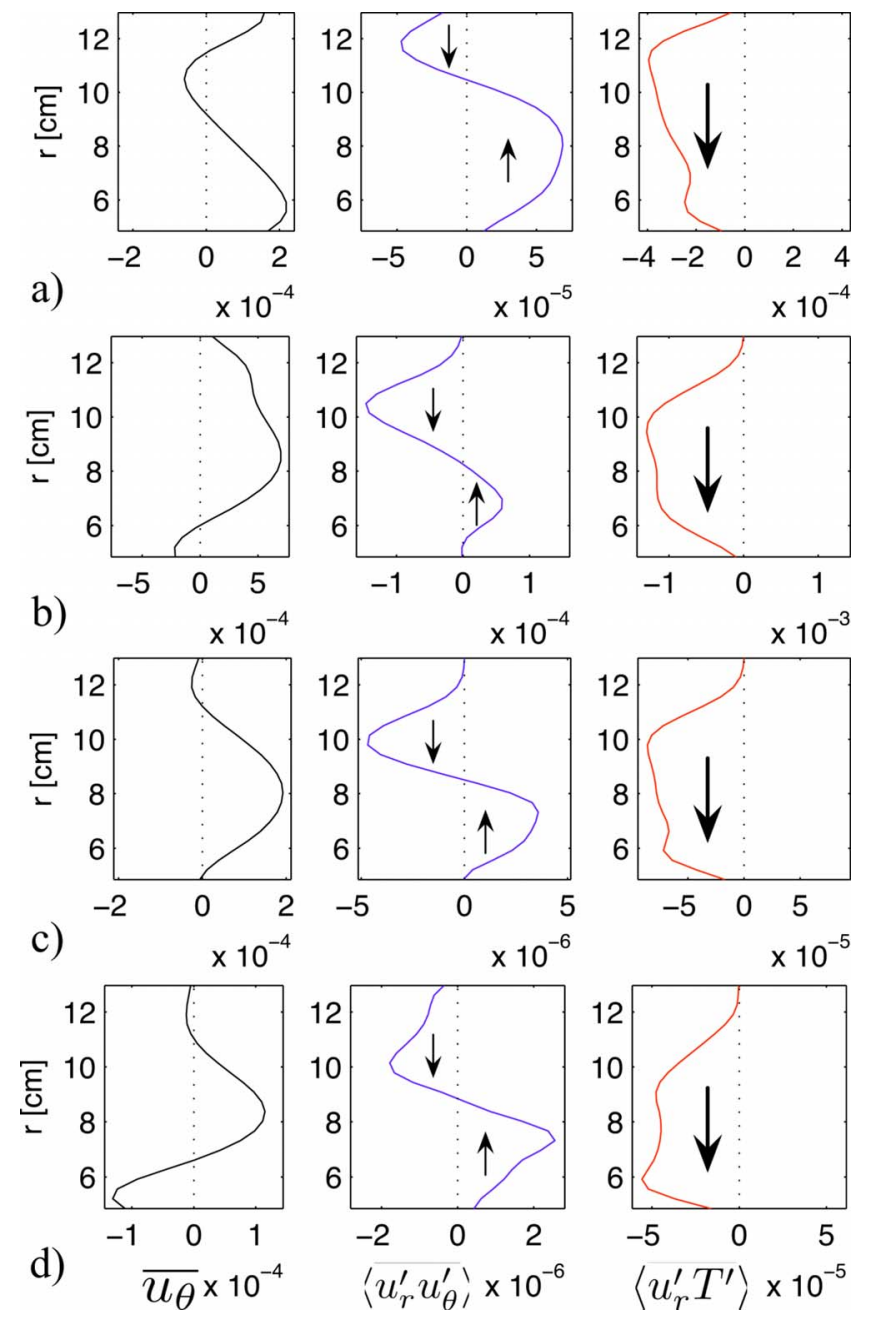

FIG. 7. (Color online) Time and zonal averaged zonal velocities $\bar{u}_{\theta}$, eddy momentum flux $\left\langle\overline{u_{r}^{\prime} u_{\theta}^{\prime}}\right\rangle$ (center), and approximate eddy heat flux $\left.\overline{\left\langle u_{r}^{\prime} T^{\prime}\right.}\right\rangle$ (right) for the same experiments as in Fig. 6.

fields by computing the inverse Laplacian $\psi^{\prime}=\nabla^{-2} \zeta^{\prime}$ with boundary conditions $\left.\psi^{\prime}\right|_{r=a, b}=0$. The calculation was performed using standard matrix inversion algorithms from the MATLAB software package.

By the definition of geostrophic streamfunction $\psi^{\prime} \equiv p^{\prime} / 2 \Omega \rho_{0}$, the hydrostatic approximation $d p^{\prime}=-g \rho^{\prime} d z$ and the linearized temperature-density relationship $\rho^{\prime}=-\rho_{0} \alpha T^{\prime}$, the approximate relationship between eddy temperature and streamfunction can be written as

$$
T^{\prime}=+\frac{2 \Omega}{g \alpha} \frac{\partial \psi^{\prime}}{\partial z} .
$$

For this analysis, Eq. (5) was converted to finite difference form, and $T^{\prime}$ estimated at intermediate depths from the multilevel streamfunction data. The eddy heat flux $\overline{\left\langle u_{r}^{\prime} T^{\prime}\right\rangle}$ was then evaluated at mid-depth via interpolation. In Fig. 7, time and zonally averaged eddy heat and momentum fluxes at mid-depth are plotted beside the time-averaged zonal flow. In all cases shown, the vertically averaged quantities were similar to those at mid-depth.

First, note that for all experiments $\left\langle\overline{\left\langle u_{r}^{\prime} T^{\prime}\right.}\right\rangle$ is negative, indicating that the eddies are always transporting heat from 
larger to smaller radii. This is expected as the radial temperature gradient is the only energy source in the experiment. Indeed, in a sense the differentially heated rotating annulus can be regarded as a heat engine, creating jet and eddy motion through the conversion of thermal to kinetic energy.

In Figs. 7(b)-7(d), the eddy momentum flux $\left\langle\overline{\left.u_{r}^{\prime} u_{\theta}^{\prime}\right\rangle}\right.$ is converging onto same-sign zonal flow, indicating that eddies were forcing the jet in each case. However, in Fig. 7(a) (the flat boundary case) positive eddy momentum flux is converging on a negative zonal jet. This is an indication that the zonal flow may have been losing kinetic energy to the eddies there.

We analyzed the Rayleigh-Kuo criterion for barotropic instability, $\beta-\partial_{r}\left[r^{-1} \partial_{r}\left(r \overline{u_{\theta}} \theta^{\theta z}\right)\right] \leq 0$, with $\overline{u_{\theta}} \theta z$ the zonal and vertical average of $u_{\theta}$, for all experiments, and found that it was usually satisfied in the high rotation rate flat boundary experiments, but never in the sloping ones. In Fig. 7(a), the positive momentum flux convergence was therefore most likely due to barotropic (or mixed barotropic/baroclinic) instability of the negative zonal flow.

In contrast to the jets produced in this experiment, those in the gas giant planets and also those produced in some recent large-scale convection experiments ${ }^{21}$ have been observed to persistently break the Rayleigh-Kuo stability criterion. Galperin et al. ${ }^{10}$ discussed the difference between stable and unstable flow regimes within the context of purely 2D barotropic theory. In the stable case, which they called frictionally dominated flow, they argued that Ekman and viscous damping act to damp the jets before they can intensify enough to break the Rayleigh-Kuo criterion.

More sophisticated eddy-mean analyses of flows with nontrivial vertical structure take eddy heat effects into account via the Eliassen-Palm (EP) flux formalism. ${ }^{22}$ In a cylindrical coordinate system, with increasing radius equivalent to a "southward" direction, the EP flux divergence is defined as

$$
\begin{aligned}
\nabla_{m} \cdot \mathbf{F} & =r^{-1} \partial_{r}\left(r F_{r}\right)+\partial_{z} F_{z} \\
& =-r^{-1} \partial_{r}\left(r\left\langle\overline{u_{r}^{\prime} u_{\theta}^{\prime}}\right)\right)-\partial_{z}\left[\left(f_{0} / d_{z} T_{0}\right)\left\langle\overline{u_{r}^{\prime} T^{\prime}}\right\rangle\right] .
\end{aligned}
$$

In the quasigeostrophic limit, Eq. (6) is equivalent to the radial flux of eddy potential vorticity $q^{\prime}$,

$$
\nabla_{m} \cdot \mathbf{F}=\left\langle\overline{u_{r}^{\prime} q^{\prime}}\right\rangle .
$$

As has been mentioned, it was not possible to collect temperature information directly for these experiments, so here the vertical temperature gradient $d_{z} T_{0}$ is replaced with the (larger) approximate value $\Delta T / d$. The vertical divergence component $\partial_{z} F_{z}$ is therefore likely to be slightly underestimated in this analysis.

In Fig. 8, the time and zonal averaged EP flux vector $\mathbf{F}$ in two experiments is plotted as a function of $r$ and $z$, with the zonal mean profile $\bar{u}_{\theta}$ superimposed. In Fig. 9, the time and zonal averaged EP flux divergence components $r^{-1} \partial_{r}\left(r F_{r}\right)$ and $\partial_{z} F_{z}$ are plotted separately, for the same experiments as in Fig. 9. If the zonal flow is entirely

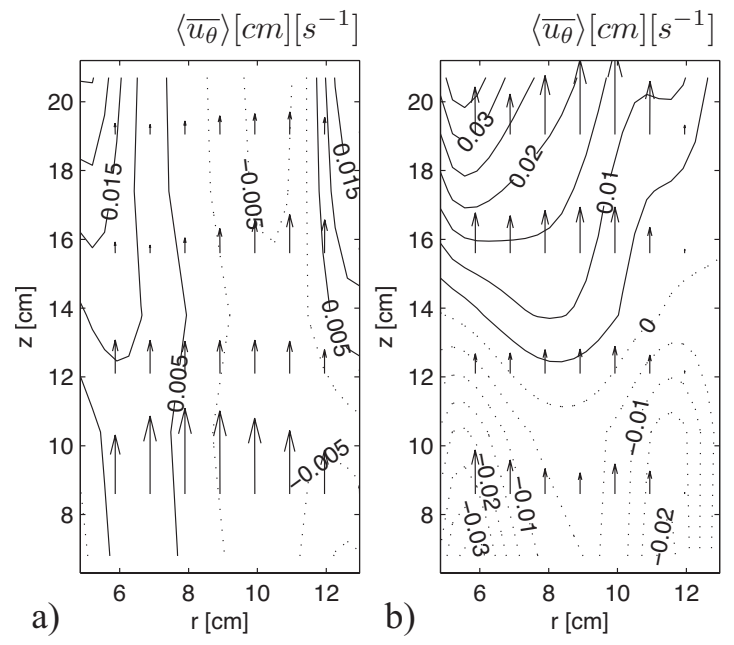

FIG. 8. Time and zonally averaged zonal velocities and EP flux vectors for the experiments corresponding to Figs. 6(a) and 6(c).

maintained by eddy motion, it is expected that $\bar{u}_{\theta}$ and $\nabla_{m} \cdot \mathbf{F}$ will be of the same sign in all regions. Conversely, if eddies are acting to weaken the zonal flow anywhere, the two quantities will be of opposing sign.
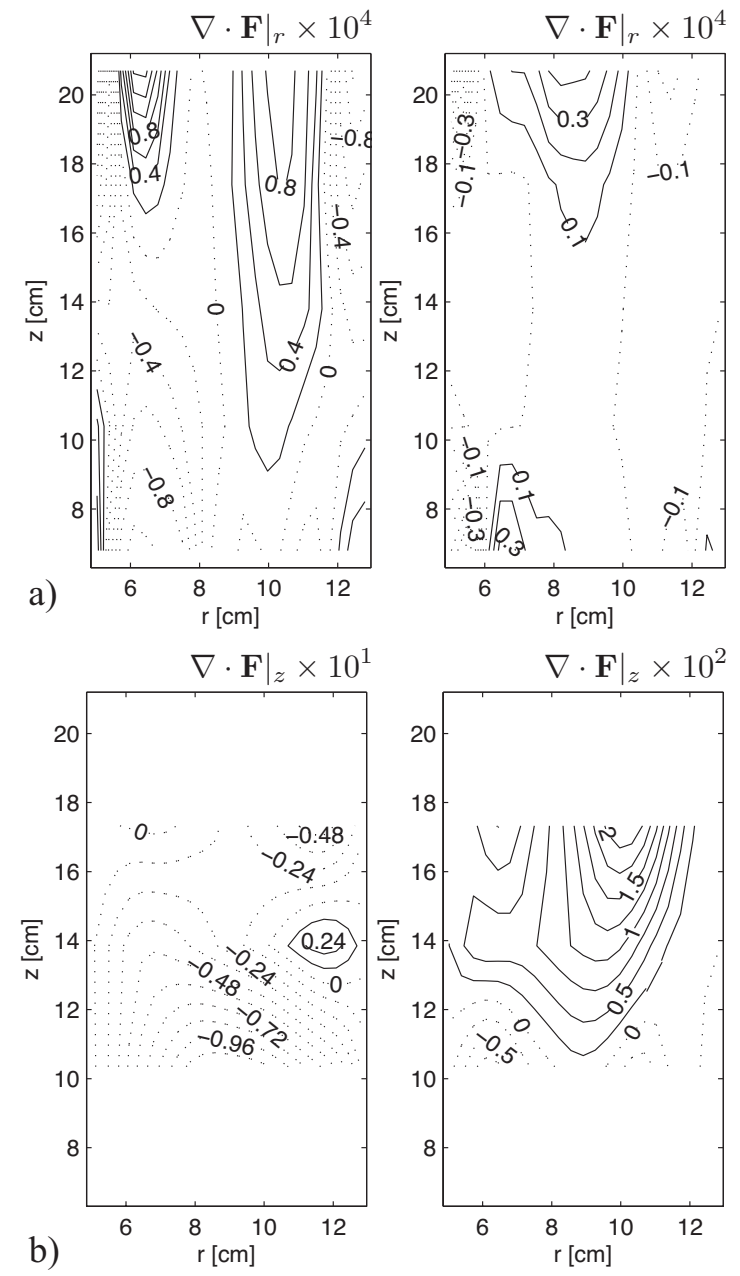

FIG. 9. Time and zonally averaged EP flux divergence components for the experiments corresponding to Figs. 6(a) and 6(c). Note that as $\left.\nabla \cdot \mathbf{F}\right|_{z}$ involves a double derivative in the vertical direction, it could only be calculated for three different depths. 
The magnitude of the $z$-component is considerably greater than that of the $r$-component in both the examples shown. It therefore appears most likely that heat effects were of greater importance to the steady-state zonal flow. Note that this was also found to be the case in some previous studies; for example, Ref. 23, in which an internally heated flat boundary numerical experiment was performed at lower Taylor numbers.

The qualitative features of the divergence fields are also of interest. In the sloping boundary plot [Fig. 9(b)], there is a clear correlation in places between $\bar{u}_{\theta}$ and both EP divergence components, $r^{-1} \partial_{r}\left(r F_{r}\right)$ and $\partial_{z} F_{z}$. This implies that both heat and momentum eddy fluxes were acting to maintain features of the observed zonal flow field.

In the flat boundary experiment, $\overline{u_{\theta}}$ was mostly anticorrelated with $r^{-1} \partial_{r}\left(r F_{r}\right)$. This implies that eddies were weakening the zonal flow, most likely through a combination of baroclinic and barotropic instability. The $\partial_{z} F_{z}$ field is more difficult to interpret, as it does not clearly relate to either the zonal flow or the eddy momentum flux divergence field. It is negative almost everywhere, peaking in the lower half of the plot. In the flat boundary experiments at lower rotation rates, we found that $\partial_{z} F_{z}$ anticorrelated with the zonal flow, implying that the initial unstable zonal flow profile was being weakened by baroclinic instability. For all high rotation rate cases, however, the $\partial_{z} F_{z}$ field took the general form seen in Fig. 9(b). It is possible that the change in $\partial_{z} F_{z}$ is linked to the transition to turbulence, in which the vertically sheared zonal flow becomes increasingly barotropic. However, without a wider range of cases to study, we could not pinpoint the physical mechanism behind the change.

As the time-averaged zonal acceleration $\partial_{t}\left\langle\bar{u}_{\theta}\right\rangle$ is expected to be small, $\nabla_{m} \cdot \mathbf{F}$ was most likely balanced by other effects in the experiments. In the sloping case, where correlation of $\left\langle\bar{u}_{\theta}\right\rangle$ with the EP flux implied eddy forcing, Ekman and direct viscous damping were probably the main effects acting to keep the zonal flow roughly constant. In the flat case, as eddies were generally acting to weaken the zonal flow, it was probably deriving most of its energy directly from the thermal forcing. However, it is possible that boundary layer effects also played a role in the overall energy budget. As we have mentioned, the vertical component of the EP divergence in the flat boundary case is rather unusual, and probably deserves further investigation.

\section{B. Spectra}

In any turbulent or nonlinear flow, the spectral view can provide great insight into the nature of the underlying dynamics. In this section, we examine $2 \mathrm{D}$ vertically averaged energy spectra for the sloping and flat boundary experiments.

Spectral analysis of an annular flow is more complicated than the rectangular channel flow case, as the correct eigenmode expansion requires combinations of Fourier and Bessel modes. It can be shown ${ }^{24}$ that any scalar $\phi$ defined in a $2 \mathrm{D}$ annular domain $b \geq r \geq a$, with boundary conditions of the form $\phi(a, \theta)=\phi(b, \theta)=0$, can be expanded in terms of the complete orthogonal basis set
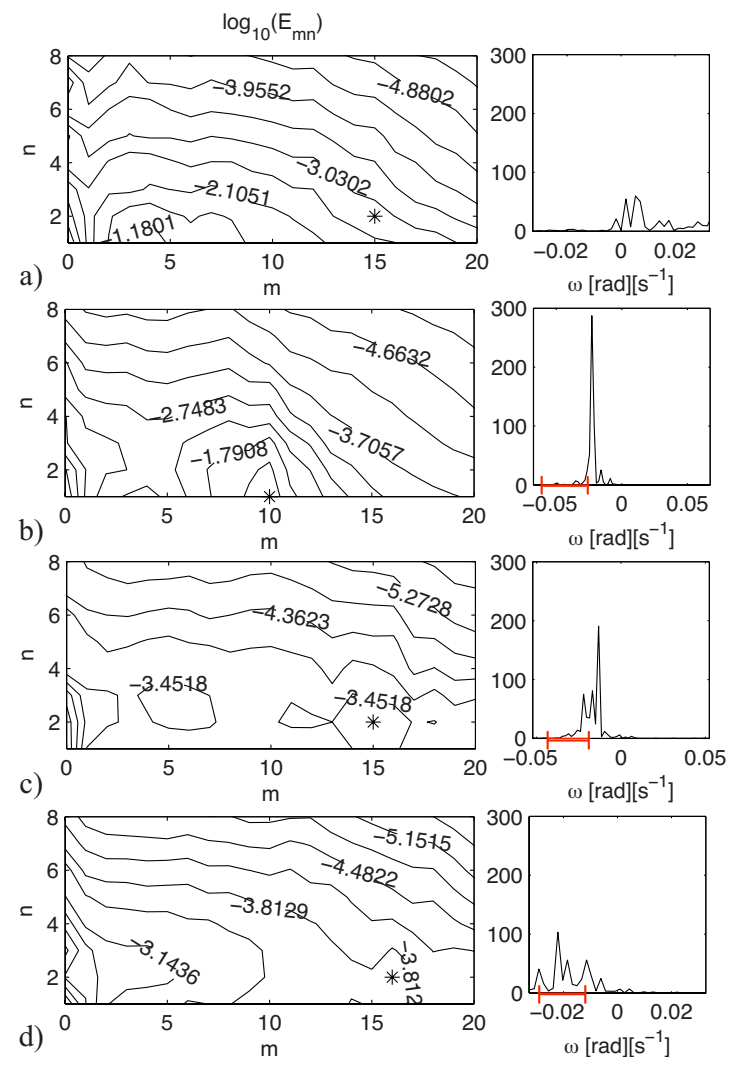

FIG. 10. (Color online) (Left) Time-averaged 2D contour plots of barotropic kinetic energy in $m, n$ spectral space for the same experiments as in Fig. 6. (Right) Temporal Fourier power spectra of spectral vorticity $\zeta_{m n}$ for the modes marked by an asterisk on the contour plots.

$$
\phi(r, \theta)=\sum_{m=-\infty}^{m=+\infty} \sum_{n=1}^{n=\infty}\left[a_{m n} J_{m}\left(\gamma_{m n} r\right)+b_{m n} Y_{m}\left(\gamma_{m n} r\right)\right] e^{i m \theta},
$$

where $J_{m}$ and $Y_{m}$ are Bessel functions of the first and second kinds and $\gamma_{m n}$ is a constant that can be determined numerically. For this analysis, we used a standard fast Fourier routine to perform the azimuthal spectral transform and then a semianalytical method to derive the radial basis modes for the laboratory annulus. ${ }^{24}$ Numerical linear algebra routines then projected experimental data onto the radial modes.

As the observed flows were, in general, quite anisotropic, it is of most interest to examine the spectra in 2D wavenumber space. In Fig. 10 (left column), we have plotted time-averaged barotropic energy spectra for several experiments as a function of azimuthal and radial wavenumbers $m$ and $n$.

In the flat boundary case, Fig. 10(a), kinetic energy decreases rapidly with wavenumber, although there are small peaks of energy at $m, n=(4,1)$. In the other plots, which are all from sloping boundary experiments, the concentration of energy in the $(0,1-3)$ zonal modes is always apparent. However, there are also peaks of energy at higher azimuthal wavenumber [approximately $m=10,15$, and 16 for Figs. 10(b)-10(d)]. These peaks are evidently due to the traveling wave structures seen in the midlevel vorticity plots.

We investigated the behavior of these waves further by Fourier transforming the relevant vorticity modes from each 
experiment in time. The chosen modes are tagged with an asterisk $(*)$ in each case, and the resulting (normalized) power spectra $\left|\zeta_{m n}(\omega)\right|^{2} / \int\left|\zeta_{m n}(\omega)\right|^{2} d \omega$ are plotted in Fig. 10 alongside the $2 \mathrm{D}$ spatial energy spectra.

While the flat boundary case has a fairly broadband distribution of frequencies at $( \pm 15,2)$, centered around $\omega=0$, the negative frequency peaks for the sloping experiments are clearly apparent. Initially, we calculated barotropic planetary wave frequencies,

$$
\omega=\frac{-\beta k}{k^{2}+l^{2}}, \quad k=\frac{m}{(b+a) / 2}, \quad l=\frac{\pi}{b-a},
$$

and compared them with the peak locations. We found that Eq. (9) significantly overestimated the magnitude of the observed values in every case studied.

Next, wave frequencies were calculated using the more general linear instability analysis of Hide and Mason, ${ }^{16}$ which includes baroclinic and Ekman effects. The predictions are indicated by the bars on the plots in Fig. 10 for the mean vertical temperature difference range $0.2 \Delta T \leq \Delta T_{z}$ $\leq 0.5 \Delta T$, in order to give a rough guide to the true analytical prediction.

Although the Hide-Mason prediction is much more accurate than the barotropic one, it still overestimated the wave frequency slightly, even with the vertical temperature gradient uncertainty taken into account. Note that it neglects the effects of annulus curvature. It is not known to what extent this affects accuracy, although errors due to other approximations (such as the neglect of direct viscous damping and Ekman layer heat transport) may well have been more significant.

At high rotation rates, the peaks become broader and more structured [Figs. 10(c) and 10(d)], almost certainly due to nonlinear mode-mode coupling. Interestingly, qualitatively similar peak broadening and shifting due to nonlinear effects have been observed by Sukoriansky et $a .^{25}$ in a numerical study of purely $2 \mathrm{D}$ planetary wave turbulence on the surface of a sphere. In the next section, we examine the exact nature of the interaction between modes using a spectral transfer calculation.

\section{Spectral energy transfer}

As a final investigation into the dynamics of the flow, we calculate the transfer rate of kinetic energy in spectral space. This can be seen as a complement to the EP flux divergence analysis carried out in Sec. III A, as our main interest is again the transfer of energy between eddies and zonal flow. However, vertically varying effects are ignored in this section. Instead, the emphasis is now on determining the extent to which energy exchange between modes is nonlocal in spectral space. Here, we assume an exchange is nonlocal if it results in a direct net transfer of energy between two modes that are not adjacent in spectral space.

To keep things simple, we only examine spectral transfer in the azimuthal direction. This removes the need to calculate triad coefficients involving Bessel functions, which simplifies the algebra considerably. Given a quasigeostrophic interior flow, the quantity
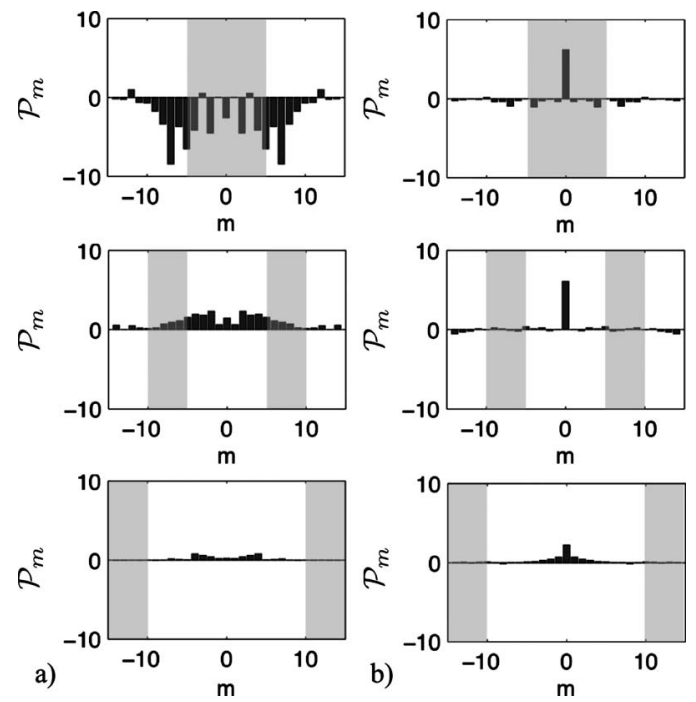

FIG. 11. Normalized azimuthal spectral energy transfer $\mathcal{P}_{m}$ as a function of azimuthal wavenumber for the experiments corresponding to Fig. 6(a) (flat boundaries) and Fig. 6(c) (sloping boundaries). For each plot, the areas marked in gray are those from which the wavenumbers $p, q$ were selected.

$$
\mathcal{P}_{m}=-\sum_{m=p+q}\left\langle T_{m p q}\right\rangle
$$

is the time and radius averaged rate of energy transfer into azimuthal mode $m$ due to all barotropic nonlinear interactions. In a steady-state system, it must be balanced by nonconservative effects such as Ekman damping or by nonlinear interactions of another type, e.g., mixed barotropicbaroclinic. The result (10) is derived in full in the Appendix, where the explicit form for the spectral transfer term $T_{m p q}$ is also given. Our approach was partly inspired by Ref. 6 in which the spectral transfer from large to small wavenumbers in a numerical simulation of $2 \mathrm{D} \beta$-plane turbulence was studied.

The sum on the right hand side of Eq. (10) is the familiar one over all triads satisfying $m=p+q$. It is too complex and time consuming to study the set of all possible triad interactions, so we chose to examine Eq. (10) for certain restricted subsets of wavenumbers. First, we focus on a subset of interactions that can cause nonlocal transfer of energy.

In Fig. $11, \mathcal{P}_{m}$ is shown for the domain $-15<m<15$, but with $p$ and $q$ restricted to the wavenumbers marked in gray for each plot. In short, Fig. 11 shows us the energy input to the entire range of wavenumbers between -15 and 15 from the wavenumbers marked in gray in each case. By definition, energy transfer to modes that are well outside the gray regions on the plots will be nonlocal. The values in the plots in Figs. 11(a) and 11(b) were normalized by constant factors for display purposes. These factors were $3.1 \times 10^{2}$ [Fig. 11(a); flat boundaries] and $2.3 \times 10^{4}$ [Fig. 11(b); sloping boundaries]. Hence the rate of energy transfer in the latter experiments was smaller by a factor of about $10^{2}$. This difference is likely to have been due to the effects of planetary wave motion in the sloping boundary system. ${ }^{8}$

In the flat boundary case, the energy transfer did not appear strongly nonlocal, broadly speaking. The set of triad interactions analyzed was causing intermediate wavenum- 

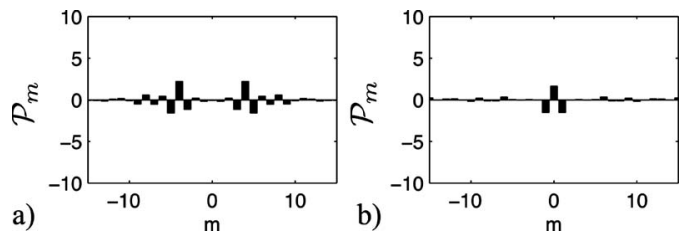

FIG. 12. Normalized azimuthal spectral energy transfer $\mathcal{P}_{m}$ as a function of azimuthal wavenumber for the experiments corresponding to Fig. 6(a) (flat boundaries) and Fig. 6(c) (sloping boundaries). For both plots, the wavenumber sum has been restricted to "local" triads only, as described in the text.

bers to lose energy to low ones, while the higher wavenumbers played a much lesser role in the exchange. In detail, however, the picture was quite complex, with significant energy exchange also occurring between the lowest azimuthal modes (top left). In addition, the highest wavenumbers were transferring some of their energy directly and nonlocally to the lowest ones.

The sloping boundary experiments exhibited qualitatively different behaviors. In almost every case, energy transfer to the zonal $m=0$ mode was found to be dominant. For the experiment shown (same rotation rate and temperature difference as flat case), substantial zonal interaction occurred even for high azimuthal wavenumbers, although we note that for the equivalent fluid B experiment (not shown), the total magnitude of energy transfer decreased more rapidly with wavenumber. In all sloping cases examined, however, the net energy transfer to the zonal mode for this subset of triads was maximum at all wavenumbers.

We also examined the energy transfer due to local triad interactions only. We defined a local triad interaction to be one in which the recipient mode $m$ is adjacent to one of the other modes $(p$ or $q)$. Because all interactions must satisfy the wavenumber rule, any local transfer of energy from, say, $p$ to $m=p \pm 1$ must involve a third wavenumber $q= \pm 1$.

In Fig. 12, we have plotted $\mathcal{P}_{m}$ for the subset of triad interactions where $m=p \pm 1$ or $q \pm 1$. Both plots were normalized by the same factors as in Fig. $12\left[3.1 \times 10^{2}\right.$ and $2.3 \times 10^{4}$ for Figs. 12(a) and 12(b), respectively]. As can be seen, the magnitude of the local transfer is fairly small compared to that of the nonlocal transfer displayed in Fig. 11. Only near $m=4$ and $m=0$ in the flat and sloping cases, respectively, is the magnitude of $\mathcal{P}_{m}$ significantly greater than zero in Fig. 12.

In itself, this result does not demonstrate that local interaction was negligible in the experimental system. It is of course possible that some modes were interacting strongly with their neighbors, but without net gain or loss of energy. Indeed, this is exactly what occurs in the inertial range of classical three-dimensional turbulence. However, an upscale transfer of energy due to purely local interaction must finish at a given wavenumber, where a net transfer of energy will be visible. The fact that $\mathcal{P}_{m}$ is small even at low wavenumbers in Fig. 12 suggests that local interactions were inputting only a small amount of energy into the zonal flow relative to the nonlocal effects shown in Fig. 11. In addition, the net transfer was negative in adjacent modes, but not at higher wavenumbers (as might be expected for a turbulent cascade).
Hence, it is likely that nonlocal spectral interaction had a more important role in the overall dynamics of the system.

It is also possible that significant local energy exchange was occurring in the barotropic-baroclinic interactions, which we have not investigated here. This seems unlikely, however, given that energy exchange between barotropic and baroclinic modes is generally most effective at the deformation wavenumber $k_{D}{ }^{1}$ As mentioned in Sec. I, it is generally argued that in a baroclinically forced turbulent flow, interactions between barotropic modes will dominate at lower wavenumbers. As significant barotropic interaction between low wavenumbers was not observed in the sloping boundary experiments, it is therefore likely that energy input to the zonal flow from baroclinic eddy effects was at least as nonlocal as in the barotropic case.

\section{CONCLUSIONS}

We investigated the behavior of a simple laboratory model of global atmospheric flow, the differentially heated rotating annulus, at high Taylor and low Hide numbers. When the top and bottom boundaries were flat, we found, as in previous studies, that the locally smooth flow observed at relatively low Taylor numbers evolved into a rapidly varying turbulent one as the rotation rate increased.

When sloping top and bottom boundaries were present, multiple jets formed at mid-depth in the fluid. An EP flux analysis showed that eddies were directly feeding momentum into the jets. Also, spectral analysis of the barotropic mode showed that eddy energy was concentrated around a definite peak wavenumber and frequency, even in the multiple jet formation regime.

The most important result of this paper, obtained through spectral transfer calculations, is that the eddies were exchanging energy directly and nonlocally in spectral space with the zonal modes. There was also a net transfer of energy due to local triad interactions, but it was weaker than the nonlocal transfer. This suggests that turbulent cascade theory, which postulates that energy exchange between local wavenumbers will dominate, is not applicable to our sloping boundary results.

We argue that nonlocal zonal flow interaction with the waves is likely to be the primary cause of jet formation in the sloping boundary experiments, but triad interactions involving infinitesimal planetary waves and a zonal flow are wellknown to be incapable of transferring energy to the zonal flow. ${ }^{26}$ It is likely, therefore, that the finite-amplitude nature of these waves is an important part of the system dynamics. Higher-order interactions of planetary waves with zonal flows have been studied by Newell ${ }^{27}$ among others, who found that energy transfer to the zonal flow is possible, but on a slower dynamical timescale. Wave-mean flow interaction theory, which in its most generalized form allows waves to be of arbitrary amplitude, also provides a framework within which jet formation by planetary wave forcing can be explained. 
It is of much interest to compare our results with the dynamics of Earth's atmosphere at midlatitudes. There, the eastward Atlantic jet stream is known to be partially maintained by a combination of eddy heat and momentum fluxes ${ }^{28}$ much like the jet produced in our sloping boundary experiments. Typically, the deformation radius in the midlatitude troposphere is of order of $1000 \mathrm{~km},{ }^{1}$ and the width of the jet stream is approximately $5000 \mathrm{~km}$. In our high rotation rate sloping boundary experiments, the horizontal buoyancy scale (4) was approximately one-tenth of the channel width. As with any laboratory study, the Ekman damping was of course greater. However, it has been suggested in some recent general circulation model studies that mean flow-eddy interactions dominate even in the Earth's atmosphere: see, for example, Refs. 29 and 30.

It is also interesting to compare these results with the zonation observed in gas giant planets. The differences in the gas giant case are somewhat greater, as interior convection, rather than baroclinic instability, may well be the dominant forcing mechanism there. Also, the observed Jovian jets are persistently barotropically unstable, whereas the ones produced by this experiment were stable. However, the basic mechanism of multiple jet formation due to correlated motion of the smaller-scale eddies does appear to be the same in both cases. ${ }^{31}$ Whether or not a spectrally local energy cascade from eddy to jet scales is occurring on the gas giant planets is, to our knowledge, still an open question.

There are several obvious possible extensions to the work presented here. One would be a more general study of geophysically relevant baroclinically forced jet regimes, with a particular focus on the nature of energy transfer between zonal and eddy modes. ${ }^{32}$ Such a study could be performed either experimentally or using a fully nonlinear numerical simulation. In the experimental case, acquisition of full three-dimensional temperature fields would be a significant advance, as it could lead to a better understanding of the thermal contribution to the EP flux (particularly in the flat boundary case).

It would also be most interesting, and perhaps simpler, to attempt to simulate the results of these experiments with a reduced theoretical or numerical model. If the dominant interaction in the sloping boundary case was between the zonal flow and planetary waves, it would appear logical in such a description to neglect wave-wave interactions as a first approximation. It is possible that a simple model based on this idea could captures the main features of the flow rather well.

\section{ACKNOWLEDGMENTS}

We thank Professor G. Vallis and a second anonymous reviewer for their insightful and constructive comments on an earlier draft of this paper. Data acquisition was performed with the use of CIV software, which is freely available online at http://www.civproject.org. This work was funded by a Natural Environment Research Council (NERC) studentship.
APPENDIX: DERIVATION OF THE ONE-DIMENSIONAL SPECTRAL ENERGY TRANSFER EQUATION

Given the quasigeostrophic approximation, ${ }^{1}$ the momentum equation in the absence of forcing and damping is simply

$$
D \mathbf{u}=\partial_{t} \mathbf{u}+\mathbf{u} \cdot \nabla \mathbf{u}=-\mathbf{f} \times \mathbf{u}_{a},
$$

where $\mathbf{u}$ is geostrophic velocity, $\mathbf{u}_{a}$ is ageostrophic velocity, and $\mathbf{u}$ only has components in the horizontal plane.

We are interested in barotropic nonlinear interactions and so ignore the right hand side of Eq. (A1). In cylindrical coordinates the remainder then becomes

$$
\begin{aligned}
& \left(\frac{\partial}{\partial t}+u_{r} \frac{\partial}{\partial r}+\frac{u_{\theta}}{r} \frac{\partial}{\partial \theta}\right) u_{r}-\frac{u_{\theta}^{2}}{r}=0, \\
& \left(\frac{\partial}{\partial t}+u_{r} \frac{\partial}{\partial r}+\frac{u_{\theta}}{r} \frac{\partial}{\partial \theta}\right) u_{\theta}+\frac{u_{r} u_{\theta}}{r}=0 .
\end{aligned}
$$

We define all variables in terms of their azimuthal Fourier coefficients,

$$
\begin{aligned}
& u_{\theta}(r, \theta, t)=\sum_{m} e^{i m \theta} u_{\theta, m}(r, t), \\
& u_{r}(r, \theta, t)=\sum_{m} e^{i m \theta} u_{r, m}(r, t) .
\end{aligned}
$$

Then substitution of Eq. (A3) into Eq. (A2) followed by a Fourier transform of the entire expression leads to

$$
\begin{aligned}
& \frac{\partial u_{r, m}}{\partial t}+\sum_{m=p+q} a_{p q}=0, \\
& \frac{\partial u_{\theta, m}}{\partial t}+\sum_{m=p+q} b_{p q}=0,
\end{aligned}
$$

with the terms $a_{p q}, b_{p q}$ defined as

$$
\begin{aligned}
& a_{p q}(r, t)=u_{r, p} u_{r, q}^{\prime}+i q \frac{u_{\theta, p} u_{r, q}}{r}-\frac{u_{\theta, p} u_{\theta, q}}{r}, \\
& b_{p q}(r, t)=u_{r, p} u_{\theta, q}^{\prime}+i q \frac{u_{\theta, p} u_{\theta, q}}{r}+\frac{u_{r, p} u_{\theta, q}}{r} .
\end{aligned}
$$

Defining semispectral energy as

$$
E_{m}(r, t)=\frac{1}{2}\left(u_{r, m} u_{r, m}^{*}+u_{\theta, m} u_{\theta, m}^{*}\right),
$$

where ${ }^{*}$ denotes complex conjugate, we can write

$$
\begin{aligned}
& \frac{\partial E_{m}(r, t)}{\partial t}=T_{m}(r, t)=-\sum_{m=p+q} T_{m p q}=0, \\
& T_{m p q}=\frac{1}{2}\left(u_{r, m}^{*} a_{p q}+u_{\theta, m}^{*} b_{p q}+\text { c.c. }\right) .
\end{aligned}
$$


Averaging in time and the radial direction

$$
\left\langle E_{m}(r, t)\right\rangle=\frac{2}{T\left(b^{2}-a^{2}\right)} \int_{t_{0}}^{t_{1}} \int_{a}^{b} E_{m}(r, t) r d r d t,
$$

where $t_{0}$ and $t_{1}$ are the starting and finishing times and $T$ $=t_{1}-t_{0}$, we arrive at the desired result,

$$
\mathcal{P}_{m}=\frac{\partial\left\langle E_{m}(r, t)\right\rangle}{\partial t}=-\sum_{m=p+q}\left\langle T_{m p q}\right\rangle
$$

In the real experiment, this quantity would have been balanced by energy loss due to Ekman and viscous damping and energy transfer due to mixed barotropic-baroclinic interactions.

${ }^{1}$ R. Salmon, Lectures on Geophysical Fluid Dynamics (Oxford University Press, New York, 1998).

${ }^{2}$ R. Fjortoft, "On the changes in the spectral distribution of kinetic energy for two-dimensional nondivergent flow," Tellus 5, 225 (1953).

${ }^{3}$ A. P. Ingersoll, P. J. Gierasch, D. Banfield, A. R. Vasavada, and The Galileo Imaging Team, "Moist convection as an energy source for the large-scale motions in Jupiter's atmosphere," Nature (London) 403, 630 (2000).

${ }^{4}$ P. B. Rhines, "Waves and turbulence on a beta-plane," J. Fluid Mech. 69, 417 (1975).

${ }^{5}$ G. P. Williams, "Planetary circulations: Barotropic representations of jovian and terrestrial turbulence," J. Atmos. Sci. 35, 1399 (1978).

${ }^{6}$ A. Chekhlov, S. Orszag, S. Sukoriansky, B. Galperin, and I. Staroselsky, "The effect of small-scale forcing on large-scale structures in twodimensional flows," Physica D 98, 321 (1996).

${ }^{7}$ H.-P. Huang and W. Robinson, "Two-dimensional turbulence and persistent zonal jets in a global barotropic model," J. Atmos. Sci. 55, 611 (1998).

${ }^{8}$ G. K. Vallis and M. E. Maltrud, "Generation of mean flows and jets on a beta plane and over topography," J. Phys. Oceanogr. 23, 1346 (1993).

${ }^{9}$ S. Sukoriansky, B. Galperin, and N. Dikovskaya, "Universal spectrum of two-dimensional turbulence on a rotating sphere and some basic features of atmospheric circulation on giant planets," Phys. Rev. Lett. 89, 124501 (2002).

${ }^{10}$ B. Galperin, S. Sukoriansky, N. Dikovskaya, P. L. Read, Y. H. Yamazaki, and R. Wordsworth, "Anisotropic turbulence and zonal jets in rotating flows with a beta-effect," Nonlinear Processes Geophys. 13, 83 (2006).

${ }^{11}$ P. L. Read, Y. H. Yamazaki, S. R. Lewis, P. D. Williams, K. MikiYamazaki, J. Sommeria, H. Didelle, and A. Fincham, "Jupiter and Saturn's convectively driven jets in the laboratory," Geophys. Res. Lett. 31, L22701, DOI: 10.1029/2004GL020106 (2004).

${ }^{12} \mathrm{R}$. Salmon, "Two-layer quasigeostrophic turbulence in a simple special case," Geophys. Astrophys. Fluid Dyn. 15, 167 (1978).
${ }^{13}$ R. L. Panetta, "Zonal jets in wide baroclinically unstable regions: Persistence and scale selection," J. Atmos. Sci. 50, 2073 (1993).

${ }^{14}$ R. Hide, "An experimental study of thermal convection in a rotating fluid," Philos. Trans. R. Soc. London, Ser. A 250, 442 (1958).

${ }^{15}$ R. Hide, S. R. Lewis, and P. L. Read, "Sloping convection: A paradigm for large-scale waves and eddies in planetary atmospheres," Chaos 4, 135 (1994).

${ }^{16}$ P. J. Mason, "Baroclinic waves in a container with sloping endwalls," Philos. Trans. R. Soc. London, Ser. A 278, 397 (1975).

${ }^{17}$ M. E. Bastin and P. L. Read, "Experiments on the structure of baroclinic waves and zonal jets in an internally heated, rotating, cylinder of fluid," Phys. Fluids 10, 374 (1998).

${ }^{18}$ A. M. Fincham and G. R. Spedding, "Low cost, high resolution DPIV for measurement of turbulent fluid flow," Exp. Fluids 23, 449 (1997).

${ }^{19}$ P. Hignett, A. A. White, R. D. Carter, W. D. N. Jackson, and R. M. Small, "A comparison of laboratory measurements and numerical simulations of baroclinic wave flows in a rotating cylindrical annulus," Q. J. R. Meteorol. Soc. 467, 131 (1985).

${ }^{20} \mathrm{~S}$. A. Condie and P. B. Rhines, "A convective model for the zonal jets in the atmospheres of Jupiter and Saturn," Nature (London) 367, 711 (1994).

${ }^{21}$ P. L. Read, Y. H. Yamazaki, S. R. Lewis, P. D. Williams, and R. D. Wordsworth, "Dynamics of convectively driven banded jets in the laboratory," J. Atmos. Sci. 64, 4031 (2007).

${ }^{22}$ D. G. Andrews, C. B. Leovy, and J. R. Holton, Middle Atmosphere Dynamics (Academic, New York, 1987).

${ }^{23}$ P. L. Read, "Finite-amplitude, neutral baroclinic eddies and mean flows in an internally heated, rotating fluid: 2 . Effects of spatially varying $N^{2}$," Dyn. Atmos. Oceans 11, 211 (1988).

${ }^{24}$ G. B. Arfken and H. J. Weber, Mathematical Methods for Physicists (Harcourt, New York, 2001).

${ }^{25}$ S. Sukoriansky, N. Dikovskaya, and B. Galperin, "On the arrest of inverse energy cascade and the Rhines scale," J. Atmos. Sci. 64, 3312 (2007).

${ }^{26}$ M. S. Longuet-Higgins and A. E. Gill, "Resonant interaction between planetary waves," Proc. R. Soc. London, Ser. A 299, 120 (1967).

${ }^{27}$ A. C. Newell, "Rossby wave packet interactions," J. Fluid Mech. 35, 255 (1969).

${ }^{28}$ S. Lee and H.-K. Kim, "The dynamical relationship between subtropical and eddy-driven jets," J. Atmos. Sci. 60, 1490 (2003).

${ }^{29}$ T. Schneider and C. C. Walker, "Self-organization of atmospheric macroturbulence into critical states of weak nonlinear eddy-eddy interactions," J. Atmos. Sci. 63, 1569 (2006).

${ }^{30}$ P. A. O'Gorman and T. Schneider, "Recovery of atmospheric flow statistics in a general circulation model without nonlinear eddy-eddy interactions," Geophys. Res. Lett. 34, L22801, DOI: 10.1029/2007GL031779 (2007).

${ }^{31}$ A. D. D. Genio, J. M. Barbara, J. Ferrier, A. P. Ingersoll, R. A. West, A. R. Vasavada, J. Spitale, and C. C. Porco, "Saturn eddy momentum fluxes and convection: First estimates from Cassini images," Icarus 189, 479 (2007).

32 "Eddy" is defined here in the standard way as a deviation from the zonal (axisymmetric) flow profile. Thus any quantity $f$ can be decomposed into zonal and eddy components: $f(r, \theta, z)=\overline{f(r, z)}+f(r, \theta, z)^{\prime}$. 